\title{
Mammographic and Ultrasonographic Evaluation of Breast Lesions with Pathological Correlation
}

\author{
Raj Bhesdadiya ${ }^{1}$, Jagruti Kalola ${ }^{2}$, Maulik Jethva ${ }^{3}$ \\ ${ }^{1}$ Resident Doctor, Department of Radio Diagnosis, PDU Medical College \& Government Hospital, Rajkot, ${ }^{2}$ Additional Professor, \\ Department of Radio Diagnosis, PDU Medical College \& Government Hospital, Rajkot, ${ }^{3}$ Associate Professor, Department of \\ Radio Diagnosis, PDU Medical College \& Government Hospital, Rajkot, India
}

Corresponding author: Dr. Raj Bhesdadiya, Umiya society, Near manokamna Temple, Dhrol-361210, Gujarat, India

DOI: http://dx.doi.org/10.21276/ijcmsr.2020.5.1.35

(c) BY-NC-ND

How to cite this article: Raj Bhesdadiya, Jagruti Kalola, Maulik Jethva. Mammographic and ultrasonographic evaluation of breast lesions with pathological correlation. International Journal of Contemporary Medicine Surgery and Radiology. 2020;5(1):A158-A161.

\section{A B S T R A C T}

Introduction: Study objectives were to determine the sensitivity \& specificity of mammography, sonomammography and both modalities together combined in assessment of breast lesions and to study role of mammography and ultrasonography in detecting breast lesions and differentiate benign and malignant lesions.

Material and methods: 50 female patients attending Department of RadioDiagnosis with palpable breast lumps over a period of 12 months were assessed using mammography, sonomammography and comparing with pathological findings.

Results: Out of 50 cases, 27 cases were diagnosed as benign, 23 were diagnosed as malignancy. Fibroadenomas were most common 15 amongst benign lesions followed by fibrocystic disease 5 and cysts 3 . There were 1 case of each lipoma \& phyllodes and 2 cases of inflammatory mastitis. Pathologically malignant lesions included atypical ductal hyperplasia (ADH), ductal carcinoma in situ (DCIS), infiltrating ductal carcinoma, lobular carcinoma, papillary carcinoma and inflammatory carcinoma. Combining the mammography and USG, sensitivity \& specificity were $92.62 \%$ and $98.12 \%$.

Conclusion: Combination of mammography and sonography plays an important role in diagnosis of palpable breast lump.

Keywords: Breast Cancer, Ultrasonography, FNAC, Ultrasonography, Mammography, Ultrasound, Fibroadenoma.

\section{INTRODUCTION}

Breast lumps are common complaints in female patients which requires early diagnosis and management. There is significant rise in the incidence of breast cancer in India. More importance is given to malignancy though benign lesions of the breast are more frequent than malignant. With the use of mammography, ultrasonography, and FNAC, the diagnosis of a benign disease can be done without surgery in the most of patients. As most of the benign lesions are not associated with an increased risk for breast cancer, unnecessary surgeries can be avoided. Delay in the diagnosis causes the malignancy to progress in advanced stage. It usually comprises of inoperable masses, metastasis which eventually results in mortality. Mammography is cost effective and widely accepted to evaluate the clinically suspicious breast lesions and screening of breast cancer. Ultrasonography is a useful adjunctive modality and helps characterizing a mammographically non-detected palpable abnormality, especially in dense breast. Sensitivity and specificity of ultrasonography or mammography is higher if ultrasonography and mammography are combined.

Study objectives were to determine the sensitivity \& specificity of mammography, sonomammography and both modalities together combined in assessment of breast lesions and to study role of mammography and ultrasonography in detecting breast lesions and differentiate benign and malignant lesions.

\section{MATERIAL AND METHODS}

This prospective study was done on 50 female patients of age 25-62 years with breast complaints who were referred to the Radiodiagnosis department of P.D.U. Medical College \& Government Hospital, Rajkot, over a period of 1 year from November 2018 to October 2019. Patients underwent routine clinical examination, Mammography and Sonography of both breasts. Ultrasonography was performed with 7.5 to $10 \mathrm{MHz}$ Linear array Transducer of Philips IU 22 ultrasound machine. Both breasts were scanned radially and by grid scanning technique with different plan. Patients were examined in supine position.

Mammography was done using a digital mammography machine. A Kilovoltage Peak ( $\mathrm{kVp}$ ) setting of 26-29 is used for average size and density breasts with focal spot of $0.4 \mathrm{~mm}$ using a target and filter of Molybdenum. Cranio-caudal (CC) and Medio-lateral(ML) views of both the breasts were done after adequate compression. Medio-lateral and oblique views of both the breasts are performed when necessary. FNAC was performed under ultrasound guidance in suspected lesions and at least two samples were taken. The results were categorized according to BIRADS (Breast 
Imaging Reporting and Data System).

\section{Inclusion criteria}

1. Women more than 25 years with palpable breast masses and complaints.

\section{Exclusion criteria}

1. Women below 25 years of age,

2. Women with advanced malignancy,

3. Fungating mass per breast,

4. Pregnant women.

5. No fine needle aspiration cytology (FNAC) available.

6. A written consent was taken either from patient or his/ her relative for FNAC.

\section{STATISTICAL ANANLYSIS}

Cross tables were done between USG (Ultrasonography), Mammography with HPE for various outcomes. Sensitivity $\&$ specificity were computed for each outcome. McNemar chi-square test was done to compare the proportions of various tests. The $\mathrm{p}$-value less than 0.05 were considered statistically significant. All the analyses were performed using SPSS version-18.

\section{RESULTS}

All symptomatic women between 25-65 years were included in the study. The mean age of women in the present study was around 40 , with most of patients $18(36 \%)$ belonging to $41-50$ years age group. $86 \%$ of the cases were in the age group of less than 50 years. Only two patients above the age of 60 years were included. (table 1)

\begin{tabular}{|l|c|c|c|}
\hline Sr No & Age in years & No. of patient & Percentage \\
\hline 1 & $25-30$ & 11 & $22 \%$ \\
\hline 2 & $31-40$ & 14 & $28 \%$ \\
\hline 3 & $41-50$ & 18 & $36 \%$ \\
\hline 4 & $51-60$ & 6 & $12 \%$ \\
\hline 5 & $>60$ & 1 & $2 \%$ \\
\hline \multicolumn{4}{|c|}{ Table-1: Age Distribution } \\
\hline
\end{tabular}

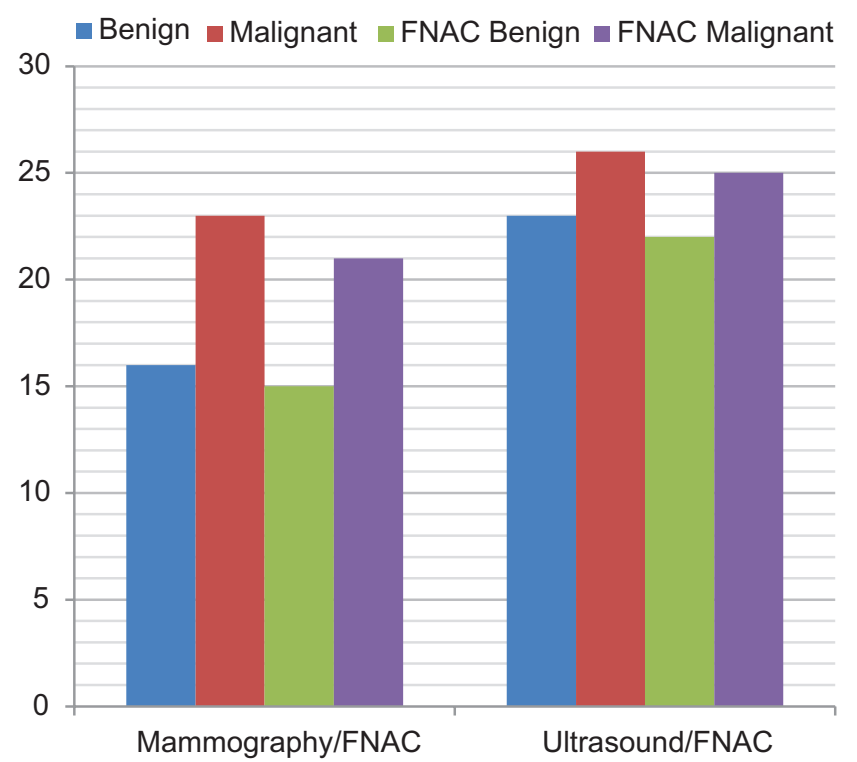

Figure-1: Comparison of mammography \& Ultrasound diagnosis with FNAC findings.

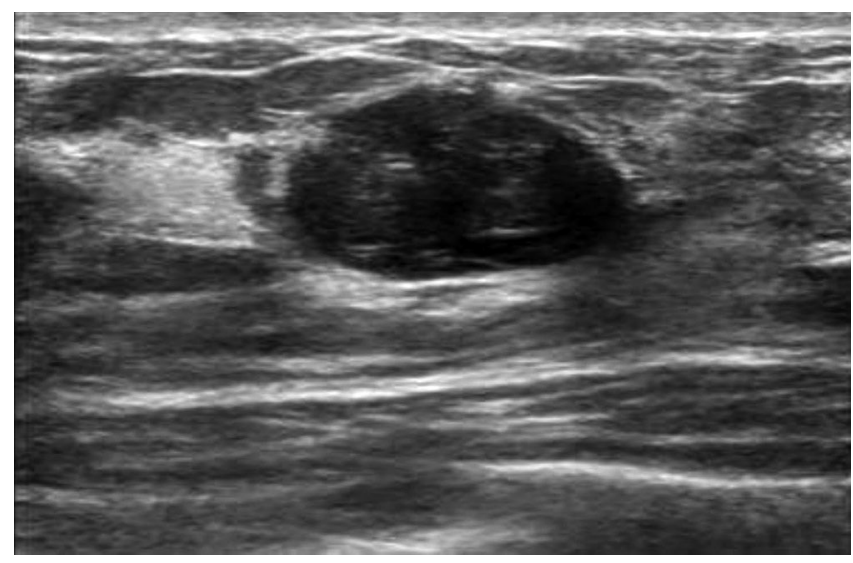

Figure-2: Ultrasound showing Fibroadenomas as mixed echogenic more hypoechoic, oval mass with smooth contour.

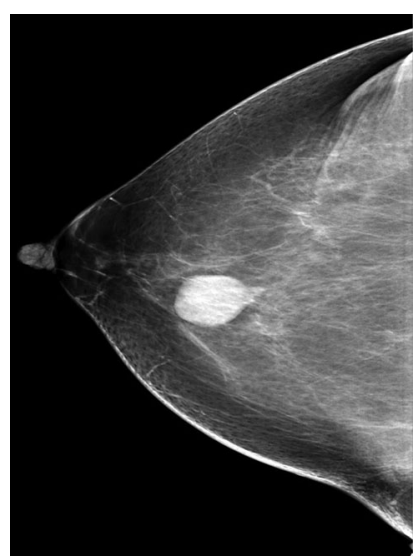

Figure-3: Mamography right breast $\mathrm{CC}$ view showing Fibroadenoma as a well circumscribed, smoothly marginated, oval mass

Out of 50 cases, 27 cases were diagnosed as benign, 23 were diagnosed as malignancy. Fibroadenomas were most common 15 amongst benign lesions followed by fibrocystic disease 5 and cysts 3 . There were 1 case of lipoma \& phyllodes and 2 cases of inflammatory mastitis. Pathologically malignant lesions included atypical ductal hyperplasia $(\mathrm{ADH})$, ductal carcinoma in situ (DCIS), infiltrating ductal carcinoma, lobular carcinoma, papillary carcinoma and inflammatory carcinoma.

Out of 50 cases, mammography could pick up 39 lesions. Amongst 39 cases, 16 were benign and 23 were malignant. In 11 cases, mammography was normal or negative for lesions. On pathological correlation, 1 out of 16 benign lesions were malignant. 21 out of 23 malignant lesions on mammography were correctly diagnosed as malignant. 1 malignant and 13 benign cases were missed on mammography. Out of 50 cases, Ultrasonography (USG) could pick up 49 lesions. Amongst 49 cases, 23 were benign and 26 were malignant. In 1 case, USG was normal or negative for lesion. On pathological correlation, 1 out of 23 benign lesions was malignant. 25 out of 26 malignant lesions were correctly diagnosed as malignant. 1 malignant case was missed on USG. Combining the mammography and USG, sensitivity $\&$ specificity were $92.62 \% \& 98.12 \%$ respectively. This study showed that there was no significant difference in sensitivity between mammography and USG $(\mathrm{p}=0.23)$. But 
there was a significant difference in mammography alone and mammography USG combination $(\mathrm{p}=0.002)$ and USG alone and combination ( $\mathrm{p}=0.0015)$. $(\mathrm{Fig}-1)$

\section{DISCUSSION}

The present study was conducted on 50 symptomatic patients with various breast related symptoms between the age of 2565 years, with a mean age of 40 years. Most of the patients (18) in 41-50 years age group. The presenting complaint in most of the patients was a mass which was palpable on selfexamination in 32 of the patients, followed by mastalgia in one or both breasts in 15 cases.

Fibroadenomas were the most common benign breast lesions in the younger females ${ }^{3}$, which presents as a freely mobile mass (breast mouse). In our study of 15 Fibroadenomas, 1 case could not be detected on mammographically dense breasts cannot not be detected because of their smaller size, in rest of the Mammograms 10 cases were labeled as benign and 2 cases as probably benign lesions due to their lobulated outlines on Mammography, Fibroadenomas appear as well circumscribed, smoothly marginated, oval masses.

Ultrasound helpful in diagnosis of all the Fibroadenomas (fig-2) as oval, round or lobulated masses with smooth contours especially where the smooth contours are hidden by adjacent dense tissue on Mammography.

In the present study Fibroadenomas are detected in 9 patients between the age of 25-30, in 4 patients between the age of $31-40$, in 1 patients between $41-50$ years and in 1 patient above the age of 50 years.

On Mammography, a cyst shows well circumscribed mass with smooth margins. cysts may be sometimes embedded in areas of dense fibrous tissue, leads to loss of definition of their margins. On Ultrasound cysts appears as oval ${ }^{4}$ or round, anechoic smooth walled lesions with posterior acoustic enhancement. Our study out of 3 cases of simple cysts, 2 Mammographs shows features of benign lesions, whereas one Mammography was normal. Ultrasound helped in detection of almost all cysts. Hence Ultrasound is the diagnostic test of choice in differentiating solid and cystic lesions of the breast. It is superior to mammography.

Fibrocystic disease with predominant cystic component appears on mammography as dense breast or illdefined masses which were read as normal, whereas on Ultrasonography multiple small cystic lesions each of less than $1.5 \mathrm{~cm}$ were are seen and which on histopathological examination appeared as adenosis and sclerosing adenosis without any cellular atypia. According to Haagenson et al Fibrocystic disease appears as dense breast tissue on mammogram whereas Ultrasonography showed only altered echotexture of parenchyma. And histopathology is more diagnostic than imaging in cases of fibroadenosis.

On Mammogram breast abscess appears as a welldefined mass or a mass with speculated borders (fig-3). On Sonography most abscess have no definite shape, have irregular contours with weak internal echoes, some abscess may have fluid-debris level or moving echoes within.

Lipoma appear as a radiolucent mass on mammography. Sometimes ultrasound couldn't be able detect a mass, as it was isoechoic to adjacent breast tissue. In our study one case which appeared as a well-defined radiolucent mass on mammography and an isoechoic mass on sonography, which was proved to be a lipoma.

\section{Malignant lesions of the breast}

Most of breast carcinomas ${ }^{8}$ are seen above age of 45 yrs. In our study carcinomas were seen above 40 years and only one patient presented below age of 40 years. In our study two patients had bilateral malignancy. Of 23 carcinoma patients 6 had axillary lymphadenopathy. Our study Mammographic sensitivity for invasive carcinoma was $90 \%$. Sensitivity of Mammography to invasive carcinoma is more because of spiculated or irregular margins with early retraction of surrounding tissue, where as lobular cancer has a poor desmoplastic reaction and diffuse distribution in contrast to invasive carcinoma hence the difference in sensitivity between these two types. Our study $92.3 \%$ of invasive carcinomas appeared as irregular or indistinct masses, which correlated well with histopathological findings similar to our study. Incidence of non-palpable malignancy in patients with palpable masses was $2.6 \%$ and median tumor size was $13.8 \mathrm{~mm}$ according to El. Rosen and Sickles. ${ }^{2}$ Hence in women with palpable breast mass it is important to screen remainder breast parenchyma for non -palpable cancer by Mammography and Sonography. ${ }^{9}$

\section{CONCLUSION}

Our study confirms the higher specificity and sensitivity for combined use of ultrasonography and mammography for differentiation of breast masses. Ultrasonography is better in differentiating solid and cystic lesions, duct ectasia, infections, pregnancy, lactation, dense breast evaluation, and real time FNAC and biopsy where as mammography is better in stereotactic biopsy, detecting microcalcifications, spiculated masses. Irregular shape, high density, spiculated margins, microcalcification, posterior acoustic shadowing, heterogeneously hypoechoic nature, internal vascularity and associated features like skin, nipple thickening and retraction favor malignancy. Oval shape, surrounding halo, wider than tall lesion, anechoic or homogenously hypoechoic lesion with posterior acoustic enhancement favor benign lesion. Ultrasonography and Mammography cannot replace each other.No single investigation is $100 \%$ accurate $^{10}$ but combination of mammography and ultrasonography can yield near better results.
Abbrevation
BIRADS (Breast Imaging Reporting and Data System)
FNAC (Fine Needle Aspiration Cytology)
Ultrasonography (USG)
Atypical ductal hyperplasia (ADH)
Ductal carcinoma in situ (DCIS)

\section{REFERENCES}

1. Breast cancer,India (Internet): Breast cancer India;20122014: Available from http://www.breastcancerindia.net

2. Sickles EA. Mammographic features of 300 consecutive non-palpable breast cancers. AJR Am J Roentgenol. 1986;146(4):661-63.

3. Cole Benglet. Sorieno R2,Kentz AB et al. Fibroadenoma of breast sonomammography Correlated with pathology 
in 122 patients. American Journal of Radiography 1983

4. Fornage BD, Toubas O, Morel M. Clinical, Mammographic and sonographic determination of preoperative breast cancer Journal of Surgical Oncology 2004;765-771

5. Stavros AT, Thickman D, Rapp CL, Dennis MA, Parker $\mathrm{SH}$, Sisney GA. Sold breast nodules; use of Sonography to distinguish between benign and malignant lesions. American journal of Radiology 2007

6. Michael A cohen, Steven J Ferlazza. Role of Sonography in Evaluation of Radial Scars of the Breast. American journal of Radiology 2000; 1075-1078.

7. Helvie MA.Adler DD, Rebner. Breast hamartomas variable mammographic appearances Radiology 1989; 170-147.

8. Hussain, Wells, Nockler PS, Lipsit ER, Blessman. Advantages and pitfalls of ultrasound in diagnosis of breast cancer. Journal of Ultrasound 2004;525-532.

9. Prasad SN, Houserkova D. A comparison of mammography and ultrasonography in the evaluation of breast masses. Biomed Pap Med Fac Univ Palacky Olomouc Czech Repub. 2007;151(2):315-22.

10. Haegenson C D; Diseases of Breast $-5^{\text {th }}$ edition. Text book of surgery,1145- 1373

Source of Support: Nil; Conflict of Interest: None

Submitted: 05-12-2019; Accepted: 07-01-2020; Published online: 28-02-2020 